Asian Pacific Journal of Tropical Biomedicine

journal homepage:www.elsevier.com/locate/apjtb

\title{
A case report of ocular toxocariasis
}

\author{
Azira NMS, Zeehaida $\mathrm{M}^{*}$ \\ Department of Medical Microbiology and Parasitology, School of Medical Sciences, Universiti Sains Malaysia, 16150, Kubang Kerian, Kelantan, Malaysia
}

\section{ARTICLE INFO}

Article history:

Received 16 February 2011

Received in revised form 28 February 2011

Accepted 13 March 2011

Available online 1 April 2011

Keywords:

Ocular toxocariasis

Ocular larva migrant

Toxocara cati

Vision

\section{ABSTRACT}

Ocular toxocariasis is prevalent among children. The symptoms and signs may mimic other ocular pathologies such as malignancies and other infectious diseases (such as toxoplasmosis and syphilis). We presented a case of progressive blurring of vision in a single eye of a 9-year-old boy. The presence of anti-toxocara antibody in serum samples helps to confirmation the diagnosis in our patient. Despite of treatment, the boy had lost his vision on the affected eye.

\section{Introduction}

Toxocariasis is the infection in humans due to Toxocara canis (T. canis) and exceptionally by Toxocara cati (T. cati). These nematodes develop the adult stage in the intestines of cats and dogs, where each female produces 200000 eggs per day. These eggs are eliminated from the body and become infecting after $2-5$ weeks. After being ingested by humans, the larvae go through the intestinal wall, migrating via the veins into the liver and the rest of the body, where they remain as larvae[1]. Clinically, two syndromes are described: visceral larva migrans (VLM) and ocular larva migrans (OLM) which, although presented as independent pathologies, are able to coexist.

The presumptive diagnosis of VLM or OLM is generally based on clinical signs, laboratory findings and a history of geophagia and contact with dogs. The clinical signs of toxocariasis are not specific and differential diagnosis includes other parasitic diseases characterized by hypereosinophilia, such as allergic reactions, asthma, other helminthiasis, such as filariasis. Peripheral eosinophilia has been constantly associated with VLM but not among patients with OLM, probably due to low larva numbers [1,2].

In Peru, a few studies were conducted to determine the toxocara $\operatorname{IgG}$ antibody using ELISA. The overall seroprevalence observed in the total population of Andrean communities from Northeast of Lima, Peru was 20.46\%[3], whereas in Amazonian city of Yurimaguas, Peru it was $35.66 \%[5]$, with a significant high proportion in children.

\footnotetext{
*Corresponding author: Zeehaida Mohamed, Department of Medical Microbiology and Parasitology, School of Medical Sciences, Universiti Sains Malaysia, 16150, Kubang Kerian, Kelantan, Malaysia.

Tel: +609-7676253

Fax: + 609-7676964

E-mail: zeehaida@kck.usm.my
}

Among the subjects with positive serology, ocular signs or symptoms ranged from $17.74 \%$ to $36.45 \%[3,4]$.

\section{Case report}

A previously healthy 9-year-old boy presented with left eye blurring of vision for 2 days prior to admission. His vision was progressively worsened. He also complained of left eye discomfort, redness and pain 1 day after the onset of illness.

However, there was no history of eye discharges. There was a history of swimming into the river. No history of trauma or foreign body to the left eye, no previous history of eye diseases. There was no history of similar conditions among family members or close friends. There was also no history of fever, skin rashes or upper respiratory tract infection symptoms.

Clinically, patient was pink, alert and conscious. Vital signs were stable and he was afebrile. On examination, left submandibular lymph node was palpable.

Other systemic review were unremarkable.

On examination of his vision, his vision of right eye was $6 / 6$ but on his left eye only perceived to light. His left eye was injected, corneal oedema, deep hypopyon and pupil mildly dilated and non reactive. However, examination of right eye was unremarkable. Scanning of the left eye showed vitreous opacity.

Left eye vitreous tap with intravitreal antibiotics with amikacin, vancomycin. Left gutt ciloxan daily and gentamycin $0.3 \%$ fortified 2 hourly. Intravenous ceftazidime $500 \mathrm{mg}$ twice daily was given for one week.

Microscopic examination of vitreous fluid showed pus cells 2 to 3 cell/field, vitreous culture did not show any bacterial growth, C-reactive protein was negative (less than $10 \mathrm{mg} / \mathrm{L}$ ), other screening tests for inflammation and infections such as antinuclear antibody (ANA), toxoplasma serology, VDRL for syphilis and HIV serology were negative. 
Toxocara IgG was detected by ELISA method.

\section{Discussion}

Human infection is due to accidental ingestion of infective eggs and tissue invasion of second stage T. cati or $T$. canis larvae. It is transmitted by contaminated food or by geophagia. Children up to 10 years are more prone to be infected for both their common geophagia[5].

OLM usually affects older children, with a mean onset age of 7.5 years and about $80 \%$ of cases less than 16 years of age. The most common symptoms and signs are strabismus, unilateral decreased vision, leukocoria, peripheral posterior pole retinal granuloma and endophthalmitis. A single eye is affected in most patients[6].

It is more frequent in children and it constitutes 1\%-2\% of uveitis in children. Toxocara should be considered as a possible causative agent of posterior and diffuse uveitis and always in the differential diagnosis of retinoblastoma. The average age of patients with ocular toxocariasis is 7.5 years (ranging from 2 to 31 years) and $80 \%$ are younger than 16 years old[9]. Three different manifestation of ocular involvement: chronic endophthalmitis, posterior granuloma and peripheral granuloma[5].

The major causes of visual acuity loss are: severe vitreitis (52.6\% of the cases), cystoid macular edema (47.4\%) and tractional retinal detachment $(36.8 \%)[8]$. The real factors that may influence the onset of a determined clinical form are unknown. It is possible that the lesions are due to a toxic or immunoallergic reaction towards larval antigens, mainly associated with larval death. The disruption occurring after larval death may determine an inflammatory reaction and granuloma formation. The associated vitreitis is usually considered as a reaction towards highly immunogenetic antigens. The severity of the disease might be related to the number of the larvae present in the eye and by the immune response of the host.

Diagnosis is based upon clinical features observed in a young patient and should be confirmed at least by the presence of specific IgG in the serum (ELISA test, 90\% specificity and $91 \%$ sensibility)[9]. Serological diagnosis of OLM is more challenging than serodiagnosis of VLM or "covert toxocariasis", because the levels of antibodies in the serum are usually low or undetectable[2,6] and eosinophilia is often absent. Even low titres of specific antibodies in the serum may be of diagnostic value but there is no consensus about the cut-off titres for diagnosis[6]. When OLM is suspected, aqueous or vitreous fluids are the best options for diagnostic samples. Even, testing of ocular fluid sample did not give absolute positive result because approximately $10 \%$ of patients with clinical signs of OLM presented negative results by ELISA method[2].

Nevertheless the absence of specific antibodies in the serum does not exclude the diagnosis of ocular toxocariasis and in such cases the presence of specific antibodies in aqueous humor demonstrate their intraocular production and confirm the diagnosis[9].

The best therapy is to prevent infection, eliminate or reduce the contact between children and contaminated environments, periodic treatment of pets, in particular lactating females. Once the infection is established, therapy should be guided according to visual acuity, severity of inflammation and irreversible ocular damage. Generally peripheral granuloma is silent or showing minimal inflammatory reaction and does not require therapy[5].

An anthelmintic therapy with either tiabendazole or diethyl carbamazepine is not worldwide accepted because of the possibility that larvae death may increase the inflammatory reaction. A steroid, either administered systemically or by periocular injections is always advisable to reduce the inflammatory reaction followed by the death of the larva or given alone to control vitreitis and the formation of vitreoretinal tractional membranes[5,6].

Sight threatening ocular inflammation requires aggressive anti-inflammatory therapy, combined with albendazole treatment $(800 \mathrm{mg} /$ day for adults and $400 \mathrm{mg} /$ day for children) for 2-4 weeks. Albendazole crosses the bloodbrain barrier and has a proven potential for killing larval stages of toxocara in the tissues of paratenic hosts[6].

Vitreoretinal surgery is useful and indicated to remove vitreous opacities and epiretinal membranes, to prevent and to treat retinal detachment. Nevertheless, in $24 \%-42 \%$ of the cases a relapse retinal detachment may occur because of a persistent post-surgical inflammatory reaction. Laser photocoagulation has a limited role and may be used to kill live and mobile larva in the retinal space when visible (under "steroid umbrella") and to treat choroidal neovascular membrane[5]. In one study which contradicted the majority, the use of anthelminthics therapy is controversial but the use of corticosteroids and vitrectomy is recommended[10].

In conclusion, ocular toxocariasis is common in developing countries but the incidence is under reported. The diagnosis requires funduscopic findings, serology and $\operatorname{IgG}$ positivity of the vitreous which may be negative in some cases. OLM may cause increase in morbidity if diagnosis is not established, leading to delay or fail to initiate prompt treatment.

\section{Conflict of interest statement}

We declare that we have no conflict of interest.

\section{References}

[1] Altcheh J, Nallar M, Conca M, Biancardi M, Freilij H. Toxocariasis: aspectos clinicos y de laboratorio en 54 pacientes. An Pediatr (Barc) 2003; 58: 425-431.

[2] Chieffi PP, dos Santos SV, de Queiroz ML, Lescano SAZ. Human toxocariasis: contribution by Brazilian researchers. Rev Inst Med Trop S Paulo 2009; 51(6): 301-308.

[3] Espinoza YA, Huapaya PE, Roldin WH, Jimenez S, Abanto EP, Rojas CA, et al. Seroprevalence of human toxocariasis in andean communities from the Northeast of Lima, Peru. Rev Inst Med Trop $S$ Paulo 2010; 52(1): 31-36.

[4] Roldan WH, Cavero YA, Espinoza YA, Jimenez S, Gutierrez CA. Human toxocariasis: a seroepidemiological survey in the Amazonian city of Yurimaguas, Peru Rev Inst Med Trop S Paulo 2010; 52(1): 37-42.

[5] Paola P. Ocular toxocariasis. Int J Med Sci 2009; 6(3): 129-130.

[6] Rubinsky-Elefant G, Hirata CE, Yamamoto JH, Ferreira MU. Human toxocariasis: diagnosis, worldwide seroprevalences and clinical expression of the systemic and ocular forms. Ann Trop Med Parasitol 2010; 104(1): 3-23.

[7] Nussenblatt RB. Toxocara canis. In: Nussenblatt RB, Whitcup S, Eds. Uveitis: Fundamentals and clinical practice. Philadelphia: Mosby; 2004, p. 244-249.

[8] Stewart JM, Cubillan LDP, Cunningham ET. Prevalence, clinical features, and causes of visual loss among patients with ocular toxocariasis. Retina 2005; 25: 1005-1013.

[9] De Visser L, Rothova A, De Boer JH, Van Loon AM, Kerkhoff FT, Canninga-van Dijk MR, et al. Diagnosis of ocular toxocariasis by establishing intraocular antibody production. Am J Ophthalmol 2007; 145: 369-374.

[10] Gomez L, Rueda T, Pulido C, Sanchez-Roman J. Ocular toxocariasis. A case report toxocariasis. Arch Soc ESP Opthalmol 2007; 83: 49-52. 